\title{
Relationship between Heart rate variability and some Pulmonary functions of handball players
}

\section{Prof. Dr/ Hamdy Abdou Abdel Wahed Asem} Dr/ El Sayed Salah El Sayed Ahmed

\section{Introduction}

Heart rate variability (HRV) is a non-invasive indicator of cardiac autonomic modulation at rest. During rhythmic exercise, global HRV decreases as a function of exercise intensity. Measures reflecting sympathy vagal interactions at rest do not behave as expected during exercise. This makes interpretation of HRV measures difficult, especially at higher exercise intensities. This problem is further confounded by the occurrence of non-neural oscillations in the highfrequency band due to increased respiratory effort.

The measurement technology for biomedical signals has developed rapidly within the past decades, and nowadays there exist many easy-to-use, affordable and mobile measurement devices for recording these signals. Preventive healthcare, personal monitoring and quantified-self are current trends where biomedical signal monitoring and analyses have a key role. For example, they can be used to objectively evaluate physical fitness, training effect and recovery time, and occupational health (stress and recovery).

Physiological tests have become the latest data that have penetrated the field of sport, especially in the field of training, and have provided effective results in the service of the scientific process and the training of sciences that have progressed and continue to achieve achievement and reach the higher levels with the completion of the physical, biological and psychological aspects to be emphasized in Exercise any sporting activity whose important role in influencing the training system should be noted. Therefore, the trainer must find the best methods and methods of measurement in order to understand some of the physiological and technical aspects associated with the procedures of using the set of standards and tests through which access to theories and 


\section{8}

scientific laws that help to achieve the best levels .(8: 19)

HRV is about variations between successive inter-beatintervals (IBIs) or RR intervals (time intervals between successive ECG R-waves). IBI varies due to ANS regulation of the sinoatrial (SA) node of the heart. ANS is divided into sympathetic and parasympathetic branches and their influences on heart rate (HR) and HRV are quite well understood. Sympathetic activity increases HR and decreases HRV, whereas parasympathetic activity decreases HR and increases HRV. The control of the autonomic output involves several interconnected areas of central nervous system, which form the so-called central autonomic network. In addition to this central control, arterial baroreceptor reflex as well as respiration are known to induce quick changes in heart rate .

\section{( $7: 22$ )}

Robergs and Roberts (2009) report that the lung supplies the body with oxygen and removes carbon dioxide, as carbon dioxide affects the acid balance in blood, and the lungs are important in regulating blood ph, Rapid changes in lung function require a sensitive control system to regulate lung function in the exchange of natural gases. Regular exercise improves lung function, although the level of improvement depends on the intensity of the training, the performance time of each training dose and the total training time. (12:307)

Adam (2011) notes that the Nervous System Autonomic (ANS) study has become more popular in recent times. It has been recognized that the change in the autonomic nervous system (ANS) may coincide with a number of hormonal changes in Over-Training (OT) and NonFunctional Over-Reaching (NFOR), it is often observed that changes in heart rhythms are a practical method for evaluating the status of the autonomic nervous system of the heart (ANS) and perhaps NFOR / OT. The results of several researchers have suggested that changes in heart rhythms can be used to guide and train elite athletes on a day to day basis. Studies on changes in heart rhythms and NFOR / OT have found mixed results, with the lowest rates in different studies. ( $2: 103$ )

Shi Jutta Mends et al. (2003) said that handball players 
cut between 6-7 $\mathrm{km}$ during the game while the distance between defense and attack and vice versa is $3-5 \mathrm{~km}$. ( 4: 163 )

The methods currently deployed to raise these levels of increasing sizes and intensities used in higher-level training loads pose some of the risks that athletes exposed to the effects of these training programs may face in order to develop, upgrade and reach the highest levels.

The improvement of the level of performance through this development in training programs and loads affects a number of biological factors, including physiological and morphological factors, but the physiological factors are at the forefront of these influences on the level of physical performance and thus the skill and the plan, which is closely related to the training loads that are exposed to it the player.

All of this requires physiological responses from the musculoskeletal system, the circulatory and respiratory systems, the player's ability to consume oxygen, delaying fatigue and the speed of recovery rates.

Richard Winsley (2002) states that the adaptive capacity of the body has facilitated the rate of response of the heartbeat to confirm the careful interaction of exposure to training pressure through paracetamobic nerve activity, which expresses the activity of the autonomic nervous system by allowing the respiratory system to respond to the challenges of these pressures. (11:328)

Guyton, A \& Hall (2011) suggests that the circulatory system serves a number of important functions in the body, most of which support other physiological devices. The circulatory system delivers oxygen and nutrients to tissues and cells in the body The transport of hormones from the endocrine to their intended receptors, removes the carbon dioxide and metabolic waste products from any cell in the body, maintains the body's temperature, and the capabilities of blood-vital organizations help regulate blood $\mathrm{pH}$ in the body. The cardiovascular system maintains appropriate fluid levels to prevent and control fluid loss and helps prevent prevention and infection. $(6: 427)$ 
The

researchers considered the importance of addressing the study by identifying the pulmonary function of the lungs and the in Heart rate variability as an indicator of physiological efficiency in a sample of handball players as one of the physiological variables affected by the results of different training loads. Change in Heart rate variability.

\section{Importance of study:}

The current study is one of the scientific attempts to study the relationship between the technical requirements of the higher levels of handball players and Heart rate variability and their pulmonary functions

Study Objective:

Identify the relationship between the rate of change in Heart rate variability and some of pulmonary functions of handball players.
Research Question:

Is there a relationship between the pulmonary functions and the Heart rate variability of the players?

\section{Methods:}

Study Methodology

The researchers used the descriptive approach

\section{SUBJECTS}

The research subjects was selected for (16) players from the professional handball league of the Aviation Club in season 2016/2017 and the measurements were made on the research sample on Sunday, 16/10/2016.

Data collection methods:

Data collection methods were selected according to the nature of the study as follows:

Measurements and devices used.

1 - Resist meter to measure the length of the poison.

2 - Electronic Weight to measure the weight in kilograms.

Table (1)

Characteristics of the subjects $N=16$

\begin{tabular}{c|c|c|c|c|c}
\hline \hline $\mathbf{N}$ & variables & Unit & mean & $\begin{array}{c}\text { standard } \\
\text { deviation }\end{array}$ & Skewness \\
\hline \hline 1 & Age & AGE & 23.8750 & 3.09570 & .564 \\
\hline 2 & Height & $\mathrm{CM}$ & 191.13 & 7.491 & .138 \\
\hline 3 & Weight & $\mathrm{Kg}$ & 97.63 & 11.063 & .032 \\
\hline \hline
\end{tabular}


Table (1) shows the homogeneity of the research sample in variables (age, height and weight), where the Skewness coefficient is limited to $( \pm 3)$.

\section{3- Measurement of Pulmonary} functions.

FitMate MED was used to measure pulmonary functions.

Table (2)

Some variables related to the pulmonary functions used in the study

\begin{tabular}{c|c|l|c}
\hline \hline $\mathbf{N}$ & Symbol & Parameter & $\begin{array}{c}\text { Unit of } \\
\text { measurement }\end{array}$ \\
\hline \hline 1 & VC & Vital capacity & $\mathrm{L}$ \\
\hline 2 & ERV & Expiratory Reserve Volume & $\mathrm{L}$ \\
\hline 3 & IRV & Inspiratory Reserve Volume & $\mathrm{L}$ \\
\hline 4 & TV & Tidal Volume & $\mathrm{L}$ \\
\hline 5 & IC & Inspiratory Capacity & $\mathrm{L}$ \\
\hline 6 & FVC & Forced Expiratory Vital Capacity & $\mathrm{L}$ \\
\hline 7 & PEF & Peak Expiratory Flow & $1 / \mathrm{sec}$ \\
\hline 8 & PIF & Peak Inspiratory Flow & $1 / \mathrm{sec}$ \\
\hline \hline
\end{tabular}

4- Measurement of Heart rate variability (HRV).

The RS800CX RUN was used to the Kubios HRV Program Pro to measure Heart rate variability and analyze the results.

Table (3)

(HRV) Variables measuring of Heart rate variability

\begin{tabular}{c|c|l}
\hline \hline $\mathbf{N}$ & Symbol & \multicolumn{1}{c}{ Parameter } \\
\hline \hline 1 & $(\mathrm{MV})$ & Average R-R interval \\
\hline 2 & SD & Standard deviation, Average R-R interval \\
\hline 3 & MSSD & $\begin{array}{l}\text { Mean Square of Differences of Successive R-R } \\
\text { Intervals }\end{array}$ \\
\hline 4 & CVr-r & Component Variance of R-R Intervals \\
\hline 5 & $($ L.E) & Difference Inspirium and Expirium heart rate \\
\hline 6 & $($ L/E\% $)$ & $\begin{array}{l}\text { Ratio maximum vers. minimum heart rate } \\
\text { during Inspirium and Expirium }\end{array}$ \\
\hline 7 & $($ Total Power ) & sums the LF and HF powers \\
\hline 8 & $\begin{array}{c}\text { Cumulative }) \\
\text { (Power }\end{array}$ & shows sum of three total power variables above \\
\hline \hline
\end{tabular}


Statistical Processes:

The researcher used the following statistical treatments using the following statistical laws:

Table (4)

Mean, standard deviation, and Skewness variance of pulmonary functions variables $N=16$

\begin{tabular}{c|c|c|c|c|c}
\hline \hline $\mathbf{N}$ & variables & $\begin{array}{c}\text { Unit of } \\
\text { measurement }\end{array}$ & Mean & $\begin{array}{c}\text { standard } \\
\text { deviation }\end{array}$ & Skewness \\
\hline \hline 1 & VC & L & 5.4044 & .72681 & $-.601-$ \\
\hline 2 & ERV & L & 1.5356 & .79652 & $-.399-$ \\
\hline 3 & IRV & L & 3.2069 & .86895 & .664 \\
\hline 4 & TV & L & .7094 & .33932 & 1.519 \\
\hline 5 & IC & L & 3.9081 & .87528 & .610 \\
\hline 6 & FVC & L & 5.1463 & .72549 & $-.358-$ \\
\hline 7 & PEF & $1 / \mathrm{sec}$ & 10.7463 & 3.02406 & 2.980 \\
\hline 8 & PIF & $1 / \mathrm{sec}$ & 5.8300 & 1.84533 & $-.271-$ \\
\hline \hline
\end{tabular}

Table (4) shows the homogeneity where the Skewness coefficient is of the research sample in limited to $( \pm 3)$

pulmonary functions variables

\section{Table (5)}

Mean and standard deviations and Skewness coefficients for of Heart rate variability $N=16$

\begin{tabular}{c|l|c|c|c}
\hline \hline $\mathbf{N}$ & \multicolumn{1}{|c|}{ Variables } & Mean & $\begin{array}{c}\text { standard } \\
\text { deviation }\end{array}$ & Skewness \\
\hline \hline 1 & $\mathrm{mv}$ & 811.5719 & 112.40592 & .437 \\
\hline 2 & $\mathrm{sd}$ & 104.9081 & 44.35715 & .617 \\
\hline 3 & $\mathrm{mssd}$ & 3171.3537 & 1617.53473 & .955 \\
\hline 4 & $\mathrm{cv} \mathrm{r}-\mathrm{r}$ & 12.1900 & 4.20615 & .219 \\
\hline 5 & ie & 26.4487 & 15.31544 & 1.976 \\
\hline 6 & 1/e & 1.4375 & .24242 & 1.028 \\
\hline 7 & total power & 4274.2563 & 4689.86950 & 2.062 \\
\hline 8 & cumulative power & 21117.6188 & 36193.97403 & 3.284 \\
\hline \hline
\end{tabular}

Table (5) shows the homogeneity of the research sample in Heart rate variability where the Skewness coefficient is limited to $( \pm 3)$ 
Table (6) 


\section{4}

Table (6) shows the matrix of correlations between measurements of pulmonary function variables and Heart rate variability, where it turns out there is a link between both air reserve d Sniffling (IRV) and exhale air reserve (ERV) when indication (.609) as well as links between the following variables function capacity Inspiration (IC) and exhale air reserve (ERV) when indication (. 623.) as well as links between the function of vital capacity (FVC) and Sniffling capacity (IC) when indication (0.507) was also a correlation between pump blood from the left atrium to the left ventricle (MV) and exhale air reserve (ERV) when indication (.506.) And also there is a link between the rate difference between spaces of different waves (CV r-r) and the standard deviation of the average squared differences between natural $\mathrm{R}-\mathrm{R}$ periods (SD), the average of the squared differences between the natural R-R periods (MSSD) at a level indication (0.812) and (0.544) respectively and existence Correlation between the difference between heart rate while you inhale and exhale (I.E) and the average of the difference between the various waves distances (CV r-r) when indication (0.535) and a significant correlation between the rate of change in heart rate during inspiration

\section{Discussion:}

Table (4) shows that pulmonary functions indicators under study are good for handball players and are close to global averages as well as the results of Egyptian handball players. The Aviation Club financiers is a large number of players of the national team

Table (5) Heart rate variability shows The results of the study that the blood flow from the left atrium to the left ventricle (MV) and the standard deviation of the mean difference between the normal RR and the average square differences between the natural RRs (MSSD) and the difference rate (CVr-r), the difference between the heartbeat during inhalation and exhalation (IE), the rate of change in heart rate during the inhalation and exhalation (L / E), the total strength of the low and high vibrations (Total 
Power) and the combined strength of the nervous system of the heart (Cumulative Power) All of these are good indicators for the average length, body weight and activity.

Abu El-Ela 'Abd ElFattah (2003) sees that the size of the heart is also related to body size. This problem is faced when we want to evaluate the physiology of athletes for the tallest "giants" or, conversely, for short stature. "Or" relative heart size "and expresses the absolute heart size in cubic centimeters. Relative heart size results from dividing the absolute size on some anthropometric indicators such as height and weight. (1: 397)

Abu El-Ela 'Abd ElFattah (2003) interval training affects the cardiovascular system during intermittent muscle work, where a hospitalization for heart rate and blood pressure between periods repeat the exercise on an individual's fitness level and environmental conditions (heat-moisture) and the duration and intensity of pregnancy Physical, in the case of light physical load performance in cold environment full hospitalization occurs during several minutes, if high load intensity or the weather warm moist it increases heart rate and not the full hospitalization during breaks interfaces, the number of repetitions to exercise the degree of intensity of physical load, which is The higher the number of iterations for intensity of physical load. (1: 423)

G.DE.H. Vito ET others (2002) suggests that the change in heart rhythms clearly reflects the activity of the sympathetic nerve and paracetamol, which control the effect of cardiac reactions resulting from the effect of exposure to stress on athletic exertion . (5: 32-38)

According to Guyton, A and Hall (2011) heart activity is largely controlled by sympathetic and parasympathetic nerves that proliferate the heart, and the amount of blood it pumps can be increased In a minute more than $100 \%$ by sympathetic alert. Conversely, this amount can be reduced to almost zero by parasympathetic sympathetic arousal: heart rate 
can be increased by up to 200 and rarely up to 250 strokes in young people The sympathetic alert also increases the strength of the heart contraction, which increases the volume of blood pumped by the heart In addition to increased ejaculation pressure, so the sympathetic alert can often increase the output of the heart by two to three times. $(6: 410)$

The higher the number of heart beats per minute, the greater the amount of blood pumped. However, there are important limits to this effect. For example, when the heart rate increases above the critical level, the strength of the heart contraction decreases, perhaps because Excessive use of the substrates in the heart muscle. In addition, the duration of the contraction between the contractions decreases so that the blood does not find the time to get enough of the atria to the ventricles. For these reasons when the heart speed is increased artificially by electrocardiogram, the heart reaches the peak of its ability to pump large amounts of blood when Its speed is between 100 and 150 feet In the minute, and on the other hand when the speed increases sympathetic alert, it reaches the peak of its ability to pump blood at a speed between 170220 beats per minute and the reason for this difference sympathetic parasympathetic alert not only increases the speed of the heart but also increases the strength of the heart at the same time Also reduces systolic contraction and gives more time to fill during extinction. (6: 121, 122) The results of Table (6) indicate the correlation matrix between pulmonary functions, which includes PIF (PEF-FVCIC-TV-IRV-ERV-VC) and Heart rate Variability (Cumulative Power- Total Power- L- E- IE- CV RRMSSD- SD- MV) where the results of the matrix table show that there are significant correlations indicating the extent to which the variables are correlated with the highest coefficient of correlation between each rate of Heart Rate Variability during (L/ E) and the difference between the heartbeat during inhalation and exhalation (IE) at (0.905), followed by the difference rate between the different wave distances ( $\mathrm{CV}$ r-r) and the 
standard deviation of the mean differences (SD) at mean level (0.812), followed by $\mathrm{L} / \mathrm{E}$ and standard deviation of mean squared differences between RR (SD) at 0.736 and the lowest The association between blood flow from the left atrium to the left ventricle (MV) and the ERV at -506- (as indicated by the above, there is no significant association between the functions of the lungs and the combined strength of the nervous system of the heart) (Cumulative Power) and the total strength of the low and high voltages of the heart (Total Power). There is also no correlation between the functions of the lungs and the deviation Special average squared differences between periods of R-R (SD), the average squared differences between the periods R-R (MSSD).

(Attachment at the end of the search)

Therefore,

the researchers see that the ability of the body to adapt is due to the rapid rate of response to the heartbeat to confirm the process of strong and accurate interaction of the pressure of training through the activity of the nerve and sympathetic and Parasympathetic expressing the activity of the nervous system self by allowing the respiratory system to respond to the response to the challenges of these external pressures, Vary in nature as a collective game for many other group games in terms of speed of rhythm and follow the various motor performances required of the players through the attacks and return quickly without interruption of the defense and throughout the time of the game all this requires the abilities of This is due to the increased activity of the Parasympathetic nerve, which in turn reduces the rate of heart rhythm during rest. Economic activity of the heart during sports with wrenches. High and during resting mode.

The researchers also see that handball is one of the activities that requires players who have morphological, physiological and physiological requirements that match the nature of motor skills in them. They are related to the performance method. They also depend on aerobic and anaerobic capacity as well as the common abilities of the 
lungs, heart, blood, blood vessels and cellular mechanisms. The muscles can contract air contractions and the maximum air capacity is equal to the maximum amount of oxygen that the organism can extract from the outside air and transfer it to the body and use it through tissue.

It also affects the functions of the heart and circulatory system and is linked to respiratory efficiency and positive responses to other vital organs of the body.

Malcolm Cook

explains that endurance means the ability to exert an effort that requires muscle contraction to produce a medium or less force of maximum force for long periods of time, requiring adaptation to heart and lung functions to meet the demands of effort.

(10: 102)

The researchers believe that this is related to the technical requirements of the functions and duties of the centers of handball players to adhere to the spaces associated with the way of play and the periods of low performance, which requires a change in the speed of movement constantly due to the philosophy of handball using advanced defense to distract the offensive thinking of the opposing team In the attempt to capture the ball and switch to a quick start to the attack, coupled with the changes to the law of handball from the rapid start of the transmission after the goal and the required continuous intervention of the nervous system, stimulating and inhibitory activity of the To match the work required by the technical functions assigned by the players and indicates the efficiency of the nervous system of the heart and the regularity of continuous stimulation and cessation in response to the change in the pace of work produced from the heart.

G.DE.H. Vito ET others (2002) suggests that the change in Heart rate variability clearly reflects the activity of the sympathetic nerve and paracetamol, which control the effect of cardiac reactions resulting from the effect of exposure to stress on athletic exertion. (5: 32-38)

Response to the question of the study 
Is there a relationship between the functions of the lungs and the change in the rhythms of the heart of the players?

1-There is a correlation between the rate of change in heart rate during inhalation and emphysema (L / E) and the difference between heartbeat during inhalation and exhalation (I.E) at (0.905).

2-There are significant associations between blood flow from the left atrium to the left ventricle (MV) and the ERV at (-506-).

3 -There is also a correlation between the difference between the heartbeat during inhalation and exhalation (I.E) and the difference between different wave distances (CV R-R) at (0.535).

\section{Conclusions}

The researchers reached the following conclusions in light of the procedures and sample of the study and methods of statistical analysis followed:

1-There is a significant correlation between pulmonary functions and the rate of change in the Heart rate variability (HRV) represented in the rate of pumping blood from the left atrium to the left ventricle (MV) and the airflow reserve (ERV).

2-The average difference between R-R and CVr-r intervals for handball players at stable limits.

3-There is also a correlation between the difference between the Heart rate variability (HRV) during inhalation and exhalation (I.E) and the difference between different wave distances (CV R-R) at (0.535).

4-There is no indication between the pulmonary functions and the combined strength of the nervous system of the heart and the overall strength of the low and high pulses of the heart.

5-There is also no correlation between pulmonary functions and the standard deviation of mean squared differences between R-R (SD) intervals, average square differences between R-Rs (MSSD).

\section{Recommendations:}

1-Focus on the functional requirements of the circulatory system and breathing necessary and appropriate for handball.

2-Conduct more similar studies on other players in different sports activities. 
3-Dependence on indicators of change in Heart rate variability (HRV) in the selection of young people because of their importance.

4-The importance of completing the study of the relationship between the change of Heart rate variability (HRV) the maximum consumption of oxygen and the threshold of anaerobic differences and other physiological and chemical variables affecting the physiological adjustment of training loads.

5-Study the relationship between the change of Heart rate variability (HRV) and genes active in the control and regulation of training loads.

\section{References}

\section{1-Abu-Ela Ahmed Abdel-}

Fattah (2003), Physiology of Training and Sports, Dar EL Faker ELA Raby Publishing, Cairo.

2-Adam L. Owen, Del P. Wong, Michel Mckenna, \& Alexandre Dellal(2011) "Heart rate response and technical comparison between Small-VS Large - sided games in elite professional Soccer, Journal of Strength and Conditioning Research .
3- Blain G, Meste O, Blain A, Bermon $S$ (2009). Timefrequency analysis of heart rate variable yields cardio locomotor coupling during dynamic cycling exercise in humans Am J Physio Heart Circ Physio 1.

4-Shi Jutta Mends, K. Chatzikotoulas, Frantisek Zahalka (2003) Optimization of the training plan of the handball game European Handball Conference .

5-G.DE.H. Vito, S.D.R. Galloway, M.A. NIMMOL, P.Maals and J.J. Mc Murray (2002) Effect of central Sympathetic inhibition on heart rate variation during steady state exercise in health humans clin physio \& fun cim, 22, pp32-38 .

6-Guyton, A and Hall (2011), J Medical Physiology, El Sevier Saunders, USA.

7-Hamdy Asem, Abd ElHalem Youssef, Khaled Ezzat (2017) ,A Comparative Study of Egyptian Football and Handball Players in Pulmonary Function and Rate of Change in Cardiac Pulses, Published Research,(TAIE) Alexandria .

8-Hamdy Asem (2008), Study of the Heart rate variability (HRV) of front and back 


\section{1}

players in the upper handball levels, published research, the First International Conference on Physical Education, Sports and Health, Kuwait, No. (19), 1-3 April 2008.

9-Hamdy Asem (2007), A study of some physiological parameters as a function of predicting the rate of Heart rate variability (HRV) of handball players "Journal of the Faculty of Physical Education Port Said, Suez Canal University.
10- Nick White Head (1994) \& Malcolm Cook Soccer training 4the ed., New York .

\section{1- Richard Winsley} (2002), Acute chronic effects of exercise on heart rate Variability in adults and children pediatric exercise science .

\section{2- Robergs, A. Roberts, S,} (2009) "Exercise Physiology" Mosby USA , III: International Information Network. 